\title{
Bestand und Turnover in der Grundsicherung
}

Die Einführung der Grundsicherung für Arbeitsuchende brachte die stärkere Forderung von Eigenbemühungen zur Arbeitsaufnahme und die Aktivierung durch den erweiterten Zugang zu den Instrumenten der Arbeitsmarktpolitik. Sie weckte hohe Erwartungen, dass Arbeitslose auf diese Weise an den Arbeitsmarkt herangeführt werden und durch das Erwerbseinkommen ihre Bedürftigkeit überwinden. Dass seitdem dauerhaft hohe Niveau an Leistungsberechtigten scheint dagegen zu signalisieren, dass diese Erwartungen enttäuscht werden. Dagegen zeigt der Beitrag auf, dass die „Starrheiten“ des Grundsicherungssystems, ebenso wie die dynamischen Prozesse darin, bisher zu wenig wahrgenommen werden.

LENA KOLLER-BÖSEL, TORSTEN LIETZMANN, HELMUT RUDOLPH

\section{Einleitung}

Die Einführung der Grundsicherung für Arbeitsuchende, das heißt des Arbeitslosengelds II (ALG II) im Sozialgesetzbuch Zweites Buch (SGB II) im Jahr 2005, stellte durch die Abkehr von statussichernder Absicherung bei Arbeitslosigkeit in der Tradition des Wohlfahrtsstaates bismarckscher Prägung einen Paradigmenwechsel in der deutschen Arbeitsmarkt- und Sozialpolitik dar. Sie wurde als bedarfsgeprüfte Flatrate-Leistung in der Erwartung angelegt, um damit Eigeninitiative und Aktivierungsforderungen zu stärken (Eichhorst et al. 2010). Diese Entwicklung ist im Rahmen des Diskurses zum Wandel vom „fürsorgenden zum aktivierenden Wohlfahrtsstaat“ zu sehen (Dingeldey 2006). Das Konzept des aktivierenden Wohlfahrtsstaates war Teil der Entwicklung eines neuen Staatsverständnisses als „dritter Weg " $z$ wischen einem neoliberal-minimalistischen und dem „alten“ sozialdemokratisch-keynesianischen Staatsverständnis (Giddens 1997, 1999). In der Arbeitsmarktpolitik zeigt es sich auf der einen Seite als Stärkung der Eigenverantwortung und daran gebundene Pflichten sowie auf der anderen Seite als Förderung der Betroffenen, um sie zu einer Arbeitsmarktteilnahme zu befähigen.

Im Sinne der neoklassischen Arbeitsmarktökonomie gelten hohe Transferleistungen als impliziter Mindestlohn und eine hohe Transferentzugsrate als wesentliche Hindernisse für einen Abbau der Arbeitslosigkeit - insbesondere im Bereich von Geringqualifizierten (Franz 2006; Sinn et al. 2002, 2006). Dadurch fehlen für Leistungsbezieher Anreize, eine Arbeit auch zu niedrigen Löhnen aufzunehmen. In dieser Argumentationslinie ist das Arbeitslosengeld-IISystem ein Schritt in die richtige Richtung, geht aber nicht weit genug. Um diesen Mechanismus zu umgehen, umfasste daher der Vorschlag einer „aktivierenden Sozialhilfe“ des ifo-Instituts (Sinn et al. 2002, 2006) ein Leistungsniveau für nicht arbeitende Leistungsbezieher, das deutlich niedriger als die ALG-II-Leistungen liegt. Dies wird ergänzt durch kommunale Beschäftigungsangebote und Lohnzuschüsse, damit für tätige Leistungsbezieher das Niveau der früheren Sozialhilfe wieder erreicht (kommunale Beschäftigung) bzw. überschritten (Lohnzuschüsse) werden kann.

Die Sichtweise, dass durch erhöhten Druck auf die Einzelnen in Richtung Erwerbsorientierung in der Grundsicherung eine höhere Dynamik und sinkende Empfängerzahlen erreicht werden können, bedarf einer Überprüfung. So erweckt das dauerhaft hohe Niveau der Bedürftigkeit den Eindruck einer überwiegend unveränderten Population von Bedürftigen, denen es nicht gelingt, ohne Sozialtransfers das sozio-kulturelle Existenzminimum zu erreichen. Es scheint, als ob sie auch durch die Förderinstrumente nicht in Arbeit und aus dem Leistungsbezug gebracht werden können. Resignativ oder moralisierend wird einem wesentlichen Teil der Leistungsempfänger häufig unterstellt, dass sie sich im Leistungsbezug „einrichten“, was aus ökonomischer Sicht bei fehlenden Arbeitsanreizen durchaus rational wäre. 
Zur Einschätzung, inwieweit sich die Erwartungen erfüllt haben, die mit dem Aktivierungs-Paradigma verbunden sind, beleuchtet die folgende Analyse die „Starrheiten“ des Grundsicherungssystems ebenso wie die dynamischen Prozesse innerhalb des Systems, die bisher weniger wahrgenommen werden. Neben der Inzidenz des SGB-II-Leistungsbezugs in der Gesamtbevölkerung und der Entwicklung der Aggregatszahlen ist vor allem wichtig, wie lange die jeweiligen Betroffenen im Leistungsbezug verweilen. Diese Betrachtung gibt Aufschluss über eine mögliche Verfestigung des Leistungsbezugs auf der individuellen Ebene und ihre etwaigen Folgen.

Gerade weil die Grundsicherung für Arbeitsuchende auf eine Erwerbsintegration ausgerichtet ist und diese fördern soll, betrachten wir auch den individuellen Arbeitsmarktstatus. Hier zeigt sich, ob eine Arbeitsmarktorientierung auf der Ebene der einzelnen Leistungsempfänger besteht und ob diese weiter gefordert werden müsste. Oder es zeigt sich, $\mathrm{ob}$ es andere Mechanismen gibt, welche die individuelle Arbeitsmotivation überlagern, und ob die Gründe für die hohen Empfängerzahlen somit eher - jenseits der einzelnen Person - in übergeordneten Bedingungen zu verorten sind. In diesem Zusammenhang ist zu berücksichtigen, dass die Grundsicherung nicht nur Arbeitslosen, sondern auch Niedrigeinkommensbeziehern und anderen Haushaltsmitgliedern das sozio-kulturelle Existenzminimum sichern soll.

\section{Entwicklung des Hilfebezugs - Bestandsstatistik}

Bei der Einführung im Januar 2005 wurden 6,12 Mio. Personen in 3,29 Mio. Bedarfsgemeinschaften in den neu geschaffenen Leistungsbereich des SGB II aufgenommen (Abbildung 1). Diese hatten zuvor überwiegend Sozialhilfe oder Arbeitslosenhilfe (Kaltenborn/Schiwarow 2006), häufig in Kombination mit Wohngeld, bezogen. Der Systemwechsel hatte nicht nur einen Einschluss-, sondern ebenso einen Ausschlussprozess von einigen Personengruppen zur Folge. Demnach erwiesen sich knapp $7 \%$ der Arbeitslosenhilfeempfänger als nicht bedürftig nach SGB II und knapp $5 \%$ der ehemaligen Sozialhilfeempfänger gingen in den Leistungsbezug nach Sozialgesetzbuch Zwölftes Buch (SGB XII) über (ebd.). Bis Ende des Jahres 2005 stieg die Anzahl der Personen auf 7,10 Mio. in 3,93 Mio. Bedarfsgemeinschaften, unter anderem weil sich für viele bisherige Wohngeldbezieher die Leistungen nach SGB II als günstiger erwiesen oder Haushalte mit Arbeitslosengeld-I-Bezug ergänzende Leistungen nach SGB II beanspruchen konnten. Im Jahresdurchschnitt 2005 bezogen im Bundesgebiet 10,1\% der Bevölkerung unter 65 Jahren Leistungen der Grundsicherung (sogenannte Hilfequote ${ }^{1}$ ).

Im Mai 2006 erreichten die Zahlen mit 7,44 Mio. Personen in 4,13 Mio. Bedarfsgemeinschaften ihren Höhepunkt. Während die Zahl der Bedarfsgemeinschaften ab diesem Zeitpunkt weitgehend kontinuierlich zurückging, schwankte die Zahl der Leistungsbezieher und stieg bis zum März 2007 wieder auf 7,42 Mio. an. Ein Grund für diese unterschiedliche Entwicklung ist, dass seit Juli 2006 Kinder im Alter von 18 bis unter 25 Jahren, die im Haushalt ihrer Eltern leben, nicht mehr als eigene Bedarfsgemeinschaft zählen, sondern Leistungen innerhalb der Bedarfsgemeinschaft ihrer Eltern erhalten.

Durch Verbesserungen der Leistungen bei Kinderzuschlag und Wohngeld in den Jahren 2008 und 2009 sank zwar die Zahl der Bedarfsgemeinschaften. Infolge der Finanzmarktund Wirtschaftskrise stiegen jedoch die Zugänge in den Leistungsbezug erneut, was die Entlastung der Grundsicherung durch die vorrangigen Sozialleistungen überlagerte, sodass sich die Zahl der Bedarfsgemeinschaften kaum veränderte. Seit Anfang 2010 ist in Deutschland ein Rückgang der Hilfebedürftigkeit nach SGB II zu beobachten. Bis Dezember 2013 sank die Zahl der Bedarfsgemeinschaften auf 3,28 Mio. mit 6,04 Mio. Personen, die Hilfequote betrug nur mehr 9,5\% (Statistik der Bundesagentur für Arbeit 2014a).

Diese Entwicklung legt zunächst die Interpretation nahe, dass aufgrund des anhaltend hohen Niveaus im Bereich der Bedürftigkeit kaum Bewegungen zu verzeichnen sind und ein relativ unveränderter Personenkreis in Bedürftigkeit verbleibt. Diese Sichtweise wurde von der dynamischen Armutsforschung für Einkommensarmut und Sozialhilfebezug in Deutschland widerlegt (Habich et al. 1991; Leibfried et al. 1995). Im Folgenden soll gezeigt werden, dass dies auch im neuen Grundsicherungssystem zutrifft und trotz gleichbleibenden Niveaus nicht immer dieselben Personen - und diese dann auch nicht dauerhaft - bedürftig bleiben.

\section{Dynamische Aspekte des Leistungsbezugs}

Neben der Entwicklung der Bestandszahlen als Maß der Hilfebedürftigkeit ist für die Betroffenen und für die Bewertung des Erfolgs von Aktivierungsbemühungen von Bedeutung, ob die Leistungen der Grundsicherung nur vorübergehend oder dauerhaft in Anspruch genommen werden müssen. So dürften bei einer kurzen Verweildauer im Leistungsbezug auch die sozialen Folgewirkungen einer vorübergehenden Armutslage geringer ausfallen. Je länger der Bezug andauert, desto eher kumulieren sich finanzielle Engpässe zu einem sinkenden Lebensstandard (Christoph/ Lietzmann 2013) und desto größer ist die Gefahr einer sozialen Abwärtsspirale. Ein hoher Anteil dauerhaft Bedürftiger stellt die Politik daher vor andere Herausforderungen als eine hohe Fluktuation im Leistungsbezug.

1 Die Hilfequote SGB II drückt den Anteil der Personen im SGB-II-Leistungsbezug in Prozent der Bevölkerung unter 65 Jahren aus. 


\subsection{Turnover}

Die Überwindung der Hilfebedürftigkeit verläuft in den meisten Fällen eher langsam. Abbildung 2 zeigt, wie sich der Monatsbestand der Personen in der Grundsicherung durch $\mathrm{Zu}$ - und Abgänge neu zusammensetzt. Die Darstellung bezieht sich dabei jeweils nur auf durchgehende Leistungsperioden ohne Unterbrechung. Tritt eine Person vorüberge-

ABB. 1

\section{Personen und Bedarfsgemeinschaften in der Grundsicherung 2005 - 2013}



Quelle: Statistik der Bundesagentur für Arbeit (2014b); Darstellung der Autoren. hend (für mindestens einen Kalendermonat) aus dem Leistungsbezug aus, wird der erneute Bezug als neue Leistungsperiode gewertet. ${ }^{2}$

Von den 6,12 Mio. Personen, die bei Einführung des SGB II im Januar 2005 bedürftig waren (Abbildung 2, linker Rand), verblieben bis zum Jahresende 2012 1,35 Mio. ununterbrochen im Leistungsbezug (hellblau gefärbter Bereich). Damit waren $22 \%$ des Ausgangsbestandes für mindestens acht Jahre im Bezug. Die übrigen 4,77 Mio. Personen (dunkelgrauer Bereich) konnten ihre Bedürftigkeit beenden oder zumindest zeitweise unterbrechen, wobei die Mehrheit davon (3,41 Mio. Personen) mindestens ein Jahr durchgehend im Leistungsbezug verblieb. Der Abbau des Anfangsbestandes verläuft zwar kontinuierlich, aber eher langsam. Bis etwa zweieinhalb Jahre nach Einführung der Grundsicherung stellen die Personen, die im Januar 2005 in den Bezug kamen, die Mehrheit des jeweiligen Monatsbestandes.

Neben den Austritten der Personen des Anfangsbestandes kommt es zu neuen Eintritten in den Leistungsbezug (hellgrauer und dunkelblauer Bereich). Am rechten Rand der Abbildung 2 ist der Stand von Dezember 2012 mit 6,04 Mio. Personen abgetragen. Zu jenen 1,35 Mio. Personen, die seit 2005 ununterbrochen auf Hilfeleistungen angewiesen waren, haben seit Anfang 2005 weitere 4,69 Mio Personen die Grundsicherung in Anspruch genommen und sich seit ihrem Zugang nicht aus der Hilfebedürftigkeit lösen können (hellgrauer Bereich). Von diesen bezogen 3,31 Mio. Personen bereits seit zwölf Monaten oder länger Grundsicherungsleistungen. Der dunkelblaue Bereich zeigt Personen, die nach Januar 2005 in den Leistungsbezug eingetreten sind und vor Ende 2012 die Bedürftigkeit wieder überwinden konnten.

\subsection{Dauer des Leistungsbezugs}

Aus Abbildung 2 lässt sich bereits erkennen, dass lange Leistungsperioden von mehr als zwölf Monaten den Hilfebezug prägen. Ergänzend ist in Tabelle 1 für alle zwischen Januar 2005 und Dezember 2012 begonnenen Leistungsbezugsepisoden die jeweilige Dauer abgebildet. Es wird dabei unterschieden, ob die Episode vollständig beobachtet werden

2 Datengrundlage der Längsschnitt- und Fluktuationsuntersuchungen ist das Administrative Panel SGB II (AdminP) des Instituts für Arbeitsmarkt- und Berufsforschung (IAB). Es handelt sich um eine $10 \%$-Stichprobe von Bedarfsgemeinschaften mit Leistungsbezug nach dem SGB II. Die Daten stammen aus dem Fachverfahren zur Leistungsgewährung in der Grundsicherung nach SGB II der Bundesagentur für Arbeit und der zugelassenen kommunalen Träger. Der Leistungsbezug von Bedarfsgemeinschaften und ihren Mitgliedern wird im AdminP so abgebildet, dass Leistungsperioden im zeitlichen Verlauf dargestellt werden können. Das AdminP wurde mit Einführung des SGB II im Januar 2005 begonnen. In der für die Analysen verwendeten Version reichen die Leistungsbiografien bis Dezember 2012. Weitere Informationen zum AdminP finden sich in Rudolph et al. (2013). 
konnte oder eine Zensierung vorliegt. Letzteres ist dann der Fall, wenn der Bezug im Dezember 2012 noch anhielt und damit unbekannt ist, wie lange diese Episode insgesamt noch andauern wird (Rechtszensierung) oder der Bezug im Januar 2005 begonnen wurde (Linkszensierung) und damit unbekannt bleibt, ob und wie lange Leistungen der Vorgängersysteme bezogen wurden.

Von allen Episoden betrug in $48 \%$ der Fälle die (bisherige) Dauer des Leistungsbezugs weniger als ein Jahr, $52 \%$ der Perioden dauerten ein Jahr oder länger. Im Falle der vollständig beobachteten Leistungsperioden (ohne Zensierungen) war der Anteil des Kurzzeitbezugs (weniger als ein Jahr) höher, da die am Datenrand noch andauernden Perioden nicht mit betrachtet werden. Die Analyse der Verweildauer im SGB-II-Bezug von Zugangskohorten hat gezeigt, dass etwas mehr als die Hälfte der Bedarfsgemeinschaften innerhalb eines Jahres den Leistungsbezug wieder verlassen hat (Graf/Rudolph 2009).

Somit ist innerhalb des Leistungsbezugs also durchaus Dynamik zu erkennen, die allerdings ungleich verteilt ist. Trotz des auf individueller Ebene relativ häufigen Auftretens von kürzeren Leistungsepisoden ist der monatliche Bestand von Langzeitbeziehern geprägt. In Tabelle 2 ist ausgewiesen, seit wie vielen Monaten die Personen, die im Dezember des jeweiligen Jahres Grundsicherungsleistungen bezogen haben, bereits ununterbrochen im SGB-II-Bezug waren. ${ }^{3}$ Ein Drittel der 7,2 Mio. Leistungsbezieher vom Dezember 2005 war seit weniger als zwölf Monaten, zwei Drittel seit zwölf Monaten, also seit der Einführung des SGB II im Januar 2005, ununterbrochen im Leistungsbezug. Dieser Anteil sinkt in den Folgejahren auf weniger als ein Viertel und unterstreicht die Dominanz von Langzeitbezug unter den zum jeweiligen Zeitpunkt im Bezug befindlichen Personen. Im Dezember 2012 dauerte die aktuelle Bezugsperiode nur bei $23 \%$ der Bezieher weniger als ein Jahr an, bei $63 \%$ bereits zwei Jahre oder länger.

\subsection{Wiederholter Leistungsbezug}

Leistungsperioden werden jedoch häufig nur vorübergehend unterbrochen, sodass die Dauer einzelner Leistungsperioden die Belastung der Hilfebedürftigen unterschätzt. Die kumulierte Dauer aller Leistungsperioden seit Januar 2005 verstärkt die Diagnose von verbreiteten verfestigten Armutslagen (Tabelle 3): $84 \%$ der Leistungsbezieher aus dem Dezember 2012 waren seit 2005 für mindestens 24 Monate aufGrundsicherungsleistungen angewiesen. Der Anteil der Personen mit kumuliertem Langzeitbezug ist über die Jahre gestiegen, u.a. weil der Zeitraum für die Erfassung von früheren Leistungsperioden größer

3 Bei der Interpretation der Dauern im Leistungsbezug ist zu beachten, dass diese bei Auswertungen von bisherigen Dauern auf Basis von Bestandspopulationen überschätzt werden, da Langfristbezieher in diesen Beständen überrepräsentiert sind (daher auch die Abweichungen von Tabelle 1). Zum Vergleich von bisheriger Dauer und Verweildauern von Zugangsstichproben im SGB II siehe Graf/Rudolph (2009) und Bergdolt et al. (2013).
TABELLE 1

\section{Dauer der Episoden mit Leistungsbezug von Personen in der Grundsicherung}

Angaben in absoluten Zahlen und in Prozent

\begin{tabular}{lc|c}
\hline & Alle Episoden & $\begin{array}{c}\text { Vollständig beobachtete } \\
\text { Episoden }\end{array}$ \\
\hline weniger als 1 Jahr & $48,0 \%$ & $63,2 \%$ \\
1 bis unter 2 Jahre & $18,0 \%$ & $19,0 \%$ \\
mindestens 2 Jahre & $34,0 \%$ & $17,8 \%$ \\
\hline Total & $100,0 \%$ & $100,0 \%$ \\
Anzahl in Tsd. & 27.097 & 16.241 \\
\hline
\end{tabular}

Anmerkung: Episoden durchgehenden Leistungsbezugs im Zeitraum 01/2005 bis 12/2012; Hochrechnung auf Basis der 211 Kreise, von denen seit 2005 vollständige Meldungen vorliegen.

Quelle: Administratives Panel SGB II; Berechnungen der Autoren.

TABELLE 2

Bisherige ununterbrochene Dauer des SGB-II-Leistungsbezugs von Personen

Angaben in absoluten Zahlen und in Prozent

Bestand zum Dezember des jeweiligen Jahres

\begin{tabular}{l|c|c|c|c|c|c|c|c}
\cline { 2 - 9 } & $\mathbf{2 0 0 5}$ & $\mathbf{2 0 0 6}$ & $\mathbf{2 0 0 7}$ & $\mathbf{2 0 0 8}$ & $\mathbf{2 0 0 9}$ & $\mathbf{2 0 1 0}$ & $\mathbf{2 0 1 1}$ & $\mathbf{2 0 1 2}$ \\
\hline $\begin{array}{l}\text { Anzahl Personen } \\
\text { (in Tsd.) }\end{array}$ & 7.178 & 7.363 & 7.093 & 6.685 & 6.818 & 6.520 & 6.128 & 6.040 \\
\hline davon & $100 \%$ & $100 \%$ & $100 \%$ & $100 \%$ & $100 \%$ & $100 \%$ & $100 \%$ & $100 \%$ \\
\hline$<12$ Monate & $33 \%$ & $26 \%$ & $23 \%$ & $23 \%$ & $25 \%$ & $22 \%$ & $22 \%$ & $23 \%$ \\
\hline $12-23$ Monate & $67 \%$ & $22 \%$ & $16 \%$ & $14 \%$ & $15 \%$ & $16 \%$ & $14 \%$ & $14 \%$ \\
\hline $\begin{array}{l}24 \text { und mehr } \\
\text { Monate }\end{array}$ & $0 \%$ & $53 \%$ & $61 \%$ & $62 \%$ & $60 \%$ & $62 \%$ & $64 \%$ & $63 \%$ \\
\hline
\end{tabular}

Anmerkung: Hochrechnung auf Basis der 211 Kreise, von denen seit 2005 vollständige Meldungen vorliegen.

Quelle: Administratives Panel SGB II; Berechnungen der Autoren.

\section{TABELLE 3}

Bisherige kumulierte Dauer des SGB-II-Leistungsbezugs von Personen Angaben in absoluten Zahlen und in Prozent

Bestand zum Dezember des jeweiligen Jahres

\begin{tabular}{|c|c|c|c|c|c|c|c|c|}
\hline & & & & & & & & \\
\hline & 2005 & 2006 & 2007 & 2008 & 2009 & 2010 & 2011 & 2012 \\
\hline $\begin{array}{l}\text { Anzahl Personen } \\
\text { (inTsd.) }\end{array}$ & 7.178 & 7.363 & 7.093 & 6.685 & 6.818 & 6.520 & 6.128 & 6.040 \\
\hline davon & $100 \%$ & $100 \%$ & $100 \%$ & $100 \%$ & $100 \%$ & $100 \%$ & $100 \%$ & $100 \%$ \\
\hline$<12$ Monate & $33 \%$ & $17 \%$ & $12 \%$ & $11 \%$ & $11 \%$ & $9 \%$ & $8 \%$ & $8 \%$ \\
\hline 12-23 Monate & $67 \%$ & $30 \%$ & $16 \%$ & $12 \%$ & $11 \%$ & $10 \%$ & $8 \%$ & $8 \%$ \\
\hline $\begin{array}{l}24 \text { und mehr } \\
\text { Monate }\end{array}$ & $0 \%$ & $53 \%$ & $72 \%$ & $78 \%$ & $79 \%$ & $81 \%$ & $84 \%$ & $84 \%$ \\
\hline
\end{tabular}

Anmerkung: Hochrechnung auf Basis der 211 Kreise, von denen seit 2005 vollständige Meldungen vorliegen.

Quelle: Administratives Panel SGB II; Berechnungen der Autoren 
wird. Dennoch ist erkennbar, dass das Rückkehrrisiko groß ist und das Ende einer Leistungsperiode häufig nur eine vorübergehende Unterbrechung der Bedürftigkeit darstellt.

Dieser Befund wird durch die Betrachtung der Zugänge in den Leistungsbezug nochmals unterstrichen. Von den Personen, die im Jahr 2012 eine (erneute) Leistungsepisode begonnen haben, hatten knapp zwei Drittel bereits in den vorangegangenen Jahren Leistungen der Grundsicherung bezogen (Tabelle 4). Im Zeitverlauf seit 2007 ging die Anzahl der Zugänge in den einzelnen Jahren zurück. Dies ist unter anderem ein Ausdruck der sich seit der Wirtschaftskrise positiv entwickelnden Arbeitsmarktlage. Gleichzeitig nimmt über den betrachteten Zeitraum der Anteil der Neuzugänge (Personen ohne vorherigen Leistungsbezug) in den Leistungsbezug ab. Bei der Interpretation dieser Zahlen ist aber zu beachten, dass die Wahrscheinlichkeit dafür, dass zugehende Personen vorher bereits einmal im Leistungsbezug waren, mit zunehmender „Lebensdauer" des Systems steigt. Festzuhalten bleibt dennoch, dass Zugänge nach vorherigem Leistungsbezug dafür sprechen, dass Ausstiege aus dem Bezug nicht immer stabil sind.

Neben den seit 2005 durchgängig Bedürftigen gibt es also eine weitere bedeutende Gruppe von Personen, die sich nur vorübergehend aus dem Leistungsbezug lösen können und wiederholt Leistungen in Anspruch nehmen. Für fast zwei Drittel der Grundsicherungsbezieher stellte sich die vorherige Beendigung des Leistungsbezug nur als Unterbrechung der Bedürftigkeit dar, die bereits häufig innerhalb weniger Monate in erneuten Leistungsbezug mündete.

Gleichzeitig haben seit 2005 erheblich mehr Menschen Erfahrung mit den Leistungen der Grundsicherung gemacht, als die Bestandszahlen vermuten lassen. So haben seit Anfang 2005 insgesamt 10,2 Mio. verschiedene Bedarfsgemeinschaften und ca. 15,3 Mio. verschiedene Personen zumindest zeitweilig Leistungen bezogen. Das soziale Netz der Grundsicherung hat in den Jahren von 2005 bis 2012 also mehr als doppelt so viele verschiedene Personen aufgefangen als zuletzt pro Monat Leistungen erhalten haben.

\section{Arbeitsmarktbeteiligung von SGB-II-Leistungsbeziehern}

Die Erwartung an die Grundsicherung war, durch Vermittlung und arbeitsmarkt- sowie sozialpolitische Förderung die Leistungsempfänger in Arbeit zu bringen und ihnen damit zu einem existenzsichernden Einkommen zu verhelfen. Einkommensanreize durch Freibeträge für Erwerbseinkommen sollen Anstrengungen für eine Erwerbsbeteiligung belohnen. Da die Grundsicherung Leistungen für ganze Bedarfsgemeinschaften erbringt, kann die Bedürftigkeit nur überwunden werden, wenn das Einkommen für alle Mitglieder erwirtschaftet werden kann. Aufgrund von Familienaufgaben einerseits, Schulbesuch und Ausbildung andererseits, ist der Kreis der erwerbsfähigen Leistungsberechtigten, die das Haushaltseinkommen verdienen können, eingeschränkt.

In der nachfolgenden Betrachtung rücken die einzelnen Leistungsempfänger und deren Potenziale für eine Erwerbstätigkeit in den Fokus. Es wird eine Differenzierung nach dem Arbeitsmarktstatus der Leistungsbezieher vorgenommen, die in einer Querschnittsbetrachtung deutlich macht, dass das Ziel der Arbeitsmarktintegration nur für einen Teil der Leistungsbezieher realistisch ist und der individuelle Arbeitsmarktstatus im Zeitverlauf durchaus variabel ist.

\subsection{Arbeitsmarktstatus}

Im Jahresdurchschnitt 2012 befanden sich 6,14 Mio. Personen im Leistungsbezug. Davon waren 1,70 Mio. (27,7\%) nicht erwerbsfähig, ${ }^{4}$ die meisten davon Kinder unter 15 Jahren (Tabelle 5). Die Grundsicherung stellt damit auch

4 Als erwerbsfähig gilt nach SGB II §8, wer zwischen 15 und 65 Jahre alt ist und mindestens drei Stunden täglich erwerbstätig sein kann.

TABELLE 4

\section{Zugänge von Personen ins SGB II (Jahressummen)}

Angaben in absoluten Zahlen und in Prozent

\begin{tabular}{|c|c|c|c|c|c|c|}
\hline & 2007 & 2008 & 2009 & 2010 & 2011 & 2012 \\
\hline Zugänge insgesamt (in Tsd.) & 2.321 & 2.211 & 2.380 & 2.194 & 1.958 & 1.908 \\
\hline \multicolumn{7}{|l|}{ Davon: } \\
\hline Vorbezug $\leq 12$ Monate & $38,3 \%$ & $40,2 \%$ & $38,7 \%$ & $36,9 \%$ & $40,3 \%$ & $37,0 \%$ \\
\hline Vorbezug $>12$ Monate & $8,9 \%$ & $15,3 \%$ & $21,3 \%$ & $24,5 \%$ & $24,6 \%$ & $27,5 \%$ \\
\hline Kein Vorbezug SGB II & $52,8 \%$ & $44,5 \%$ & $40,0 \%$ & $38,6 \%$ & $35,0 \%$ & $35,5 \%$ \\
\hline
\end{tabular}

Anmerkung: „kein Vorbezug SGB II“: Erstzugang in die Grundsicherung

"Vorbezug": Die Person hat bereits innerhalb der letzten zwölf Monate bzw. vor mehr als zwölf Monaten Grundsicherungsleistungen erhalten

und war vor dem Zugang für mindestens einen Kalendermonat ohne Leistung.

Hochrechnung auf Basis der 211 Kreise, von denen seit 2005 vollständige Meldungen vorliegen.

Quelle: Data warehouse der Bundesagentur für Arbeit; Berechnungen der Autoren. 
für Familien ein bedeutendes System der sozialen Sicherung dar. Kinder sind allerdings keine Zielgruppe für die Arbeitsförderung bzw. -vermittlung. Um ein Ende des Leistungsbezugs zu erreichen, muss ihr Bedarf durch eine Erwerbstätigkeit der Eltern mit erwirtschaftet werden.

Die übrigen 4,44 Mio. waren erwerbsfähige Leistungsberechtigte, die sich zum einen nach ihrem Arbeitslosigkeitsstatus und zum anderen hinsichtlich ihrer Erwerbstätigkeit unterscheiden lassen. 42,9\% der erwerbsfähigen Leistungsberechtigten waren arbeitslos gemeldet, ${ }^{5}$ während über die Hälfte der Erwerbsfähigen (57,1 \%) aufgrund verschiedener Aktivitäten nicht als arbeitslos registriert war. 10,4\% nahmen an arbeitsmarktpolitischen Maßnahmen teil und 14,6\% der erwerbsfähigen Leistungsempfänger waren in ungeförderter Erwerbstätigkeit beschäftigt. Nimmt man diejenigen zusammen, die arbeitslos, in Maßnahmen oder in ungeförderter Beschäftigung waren, zeigt sich, dass 67,9\% der erwerbsfähigen Leistungsberechtigten am Arbeitsmarkt aktiv oder zumindest grundsätzlich verfügbar waren. Fast ein Drittel der erwerbsfähigen Leistungsberechtigten war jedoch kurzfristig nicht am Arbeitsmarkt oder in der Arbeitsuche aktiv. Sie waren durch Schule und Ausbildung (7,2\%) oder durch Betreuungsaufgaben $(6,7 \%)$ gebunden, vorübergehend arbeitsunfähig (5,7\%) oder bereits im Vorruhestand (5,2\%).

Im unteren Teil der Tabelle 5 sind die erwerbsfähigen Leistungsberechtigten nach ihrer tatsächlichen Erwerbstätigkeit aufgeführt. Erwerbstätigkeit ist hier breiter definiert als oben und misst lediglich das Vorliegen einer Erwerbstätigkeit, unabhängig von deren Umfang (inkl. geringfügiger Beschäftigung). Insgesamt waren 2012 29,8\% der erwerbsfähigen Leistungsberechtigten erwerbstätig (sogenannte Aufstocker), also mehr als bei Betrachtung des Hauptstatus „ungeförderte Erwerbstätigkeit“. Die Differenz erklärt sich vor allem aus den geringfügig Beschäftigten (10,7\%), die als Arbeitslose, Maßnahmeteilnehmer, Schüler oder Betreuende in geringem Umfang arbeiten sowie durch die in den Maßnahmen geförderten Beschäftigten.

Die Betrachtung der verschiedenen Personengruppen im SGB-II-Leistungsbezug mit ihrem Arbeitsmarktstatus macht deutlich, dass es sich nicht nur um ein System zur Absicherung bei Arbeitslosigkeit handelt. So sind Familienangehörige (speziell Kinder) mit abgesichert sowie erwerbstätige Personen, die ein Erwerbseinkommen erzielen, das den Lebensunterhalt des Haushaltes nicht gewährleistet (vgl. Bruckmeier et al. 2013). Des Weiteren befinden sich in der Grundsicherung auch Erwerbsfähige, die für eine Aktivierung nicht direkt erreichbar sind. Die Potenziale für eine weitergehende Anwendung von Aktivierungsmaßnahmen sind somit deutlich eingeschränkt.

5 Das heißt, sie arbeiten nicht länger als 15 Stunden pro Woche. Geringfügig beschäftigte Leistungsberechtigte sind damit meist als arbeitslos registriert.

\subsection{Statusdynamik}

Vor diesem Hintergrund ist trotz eines verbreiteten Langzeitleistungsbezugs nicht auf Langzeitarbeitslosigkeit oder längerfristiger Untätigkeit auf der individuellen Ebene zu schließen. Die folgende Betrachtung beleuchtet die Stabilität von Arbeitslosigkeit und Erwerbstätigkeit im Vergleich zum Leistungsbezug. Bei andauerndem Leistungsbezug wechselt ein Teil der Leistungsbezieher zwischen Arbeitslosigkeit und Erwerbstätigkeit oder Maßnahmenteilnahme, ohne den Grundsicherungsbezug zu beenden.

Der erste Zeilenblock in Tabelle 6 gibt Kennziffern für erwerbsfähige Leistungsberechtigte an. Im Jahr 2012 haben durchschnittlich pro Monat 4,44 Mio. Erwerbsfähige SGBII-Leistungen erhalten. Aufsummiert über den Jahresverlauf waren 5,54 Mio. verschiedene Personen vom Grundsicherungsbezug betroffen. Bei einer hohen Fluktuation aus $\mathrm{Zu}$ und Abgängen steigt die Zahl der „Betroffenen“, die

\section{TABELLE 5}

\section{Personen im Leistungsbezug SGB II nach Arbeitsmarktstatus - Jahresdurchschnitt 2012}

Angaben in absoluten Zahlen und in Prozent

\begin{tabular}{|c|c|c|}
\hline & & $\begin{array}{l}\text { Anzahl } \\
\text { in Tsd. }\end{array}$ \\
\hline Bedarfsgemeinschaften & & 3.325 \\
\hline Personen/Leistungsberechtigte: & & 6.143 \\
\hline erwerbsfähig & & 4.443 \\
\hline \multirow[t]{2}{*}{ nicht erwerbsfähig } & Kinder unter 15 Jahren & 1.620 \\
\hline & sonstige ab 15 Jahren & 79 \\
\hline \multicolumn{3}{|l|}{$\begin{array}{l}\text { Erwerbsfähige } \\
\text { Leistungsberechtigte }\end{array}$} \\
\hline Anzahl & & 4.443 \\
\hline in $\%$ & & $100 \%$ \\
\hline \multirow{10}{*}{$\begin{array}{l}\text { arbeitslos } \\
\text { nicht arbeitslos }\end{array}$} & & $42,9 \%$ \\
\hline & & $57,1 \%$ \\
\hline & darunter*: & \\
\hline & in arbeitsmarktpolitischen Maßnahmen & $10,4 \%$ \\
\hline & in ungeförderter Erwerbstätigkeit & $14,6 \%$ \\
\hline & in Schule, Studium, ungeförderter Ausbildung & $7,2 \%$ \\
\hline & in Erziehung, Haushalt, Pflege & $6,7 \%$ \\
\hline & in Arbeitsunfähigkeit & $5,7 \%$ \\
\hline & in Vorruhestand & $5,2 \%$ \\
\hline & unbekannt & $5,9 \%$ \\
\hline \multirow{4}{*}{$\begin{array}{l}\text { nicht erwerbstätig } \\
\text { erwerbstätig }\end{array}$} & & $70,2 \%$ \\
\hline & & $29,8 \%$ \\
\hline & darunter: & \\
\hline & ausschließlich geringfügig beschäftigt & $10,7 \%$ \\
\hline
\end{tabular}

* Anmerkung: Die ausgewiesenen Anteile wurden aus den Grundsicherungsstatistiken der Bundesagentur für Arbeit übernommen und stimmen - aufsummiert - auch dort nicht mit dem Anteil nicht arbeitsloser erwerbsfähiger Leistungsberechtigter überein. Gleiches gilt für die zugrunde liegenden absoluten Zahlen.

Quelle: Statistik der Bundesagentur für Arbeit (2010, 2011 und 2013); Darstellung der Autoren. 
im Laufe des Jahres die Grundsicherung zumindest vorübergehend in Anspruch nehmen mussten.

Die Relation zwischen im Kalenderjahr betroffenen Personen und dem (Jahres-)Durchschnitt wird als „Betroffenheit" bezeichnet; sie bildet ein Maß für die Verteilung der Bedürftigkeit auf mehr oder weniger erwerbsfähige Leistungsbedürftige. Für 2012 ergibt sich eine Relation von Betroffenen zum Durchschnitt von $125 \%$.

Betrachtet man die Dauer des Leistungsbezugs, so ergibt sich, dass 3,32 Mio. erwerbsfähige Leistungsberechtigte während des gesamten Jahres 2012 durchgehend Leistungen bezogen haben. Die Relation zwischen im Kalenderjahr durchgehend im Bezug stehenden Personen und dem (Jahres-) Durchschnitt gibt den Grad der „Verfestigung“ an. Je höher der Anteil ist, umso größer ist das Ausmaß der Verfestigung des Leistungsbezugs bezogen auf das Kalenderjahr. Im Jahr 2012 machte der verfestigte Leistungsbezug am Jahresdurchschnitt einen Anteil von $75 \%$ aus. Aus der Differenz von betroffenen Personen zu solchen mit durchgehendem Bezug ergeben sich 2,22 Mio. erwerbsfähige Leistungsberechtigte, die durch Zugang oder Abgang im Laufe des Jahres nur zeitweilig bedürftig waren.

\section{TABELLE 6}

\section{Eckdaten zur Grundsicherung nach SGB II - 2012}

Angaben in absoluten Zahlen in Tausend und in Prozent

\begin{tabular}{l|r}
\hline Erwerbsfähige Leistungsberechtigte & \\
\hline Betroffene & 5.543 \\
\hline (Jahres)Durchschnitt & 4.443 \\
\hline Durchgehend & 3.322 \\
\hline Betroffenheit: Betroffene/Durchschnitt & $125 \%$ \\
\hline Verfestigung: durchgehend/Durchschnitt & $75 \%$ \\
\hline Arbeitslose Leistungsberechtigte & \\
\hline Betroffene & 3.508 \\
\hline (Jahres)Durchschnitt & 1.907 \\
\hline Durchgehend & 909 \\
\hline Betroffenheit: Betroffene/Durchschnitt & $184 \%$ \\
\hline Verfestigung: durchgehend/Durchschnitt & $48 \%$ \\
\hline Erwerbstätige Leistungsberechtigte & \\
\hline Betroffene & 2.159 \\
\hline (Jahres)Durchschnitt & 1.167 \\
\hline Durchgehend & 530 \\
\hline Betroffenheit: Betroffene/Durchschnitt & $185 \%$ \\
\hline Verfestigung: durchgehend/Durchschnitt & $45 \%$ \\
\hline
\end{tabular}

Anmerkung: Die Tabelle zeigt, wie sich der Jahresdurchschnittsbestand an erwerbsfähigen Leistungsberechtigten aufteilt nach Betroffenheit (wie viele verschiedene Personen?) und dauerhaftem Leistungsbezug. Bei anhaltendem Leistungsbezug findet ein Statuswechsel zwischen "arbeitslos" und "erwerbstätig" statt, da die Betroffenheit höher, der ganzjährig andauernde Status seltener ist als bei den erwerbsfähigen Leistungsberechtigten Langzeitiger Leistungsbezug ist nicht identisch mit Langzeitarbeitslosigkeit. Hochrechnung auf Basis der 211 Kreise, von denen seit 2005 vollständige Meldungen vorliegen.

Quelle: Data warehouse der Bundesagentur für Arbeit und Administratives Panel SGB II; Berechnungen der Autoren.
Im mittleren und unteren Zeilenblock in Tabelle 6 sind die gleichen Kennzahlen für arbeitslose und erwerbstätige Leistungsberechtigte dargestellt. Im Jahresverlauf 2012 waren 3,51 Mio. erwerbsfähige Leistungsberechtigte arbeitslos gemeldet. Bezogen auf den Jahresdurchschnitt von 1,91 Mio. arbeitslosen Leistungsberechtigten ergibt dies eine Betroffenheit von $184 \%$. Während des gesamten Jahres waren hingegen nur 0,91 Mio. Leistungsberechtigte durchgehend arbeitslos. Die Arbeitslosigkeit im SGB II wird also häufiger unterbrochen und ist weniger verfestigt als der Leistungsbezug selbst. Somit geht langzeitiger Leistungsbezug häufig mit Statusmobilität zwischen Arbeitslosigkeit, nicht bedarfsdeckender Erwerbstätigkeit oder anderen Lebenslagen einher.

Diese Statusmobilität zeigt sich auch bei den erwerbstätigen Leistungsberechtigten. Gemessen am Jahresdurchschnitt von 1,17 Mio. Aufstockern waren $45 \%$ ganzjährig erwerbstätig mit zusätzlichen Grundsicherungsleistungen, sodass die Zahl der Personen, die innerhalb des Jahres zumindest vorübergehend einer Beschäftigung nachgingen, den Jahresdurchschnitt um fast das Doppelte überstieg (Betroffenheit: $185 \%$ des Jahresdurchschnitts).

\section{Diskussion: Grenzen der Aktivierungspolitik}

Die Betrachtung der dynamischen Aspekte des Leistungsbezugs hat gezeigt, dass - trotz der andauernd hohen Empfängerzahlen und der zumindest im Bestand vorherrschenden Dominanz von Langzeitbezug - auf individueller Ebene durchaus Bewegung zu erkennen ist. Die einzelnen Leistungsbezugsepisoden dauern in ca. der Hälfte der Fälle weniger als zwölf Monate. In beträchtlichem Umfang ist aber ein wiederholter Leistungsbezug zu beobachten. Dies spricht für einen ersten sogenannten Drehtüreffekt: Gelingen Ausstiege aus dem Leistungsbezug, sind diese nicht immer nachhaltig. Dies kann unter anderem daran liegen, dass Beschäftigungsverhältnisse, die aus dem Bezug heraus begonnen wurden, nur in $55 \%$ der Fälle länger als sechs Monate andauern (Koller/Rudolph 2011). Bei Wegfall dieser Erwerbstätigkeit oder anderen Ereignissen im Haushaltskontext kann es zu einem erneuten Leistungsbezug kommen.

Wechselt man die Perspektive vom Leistungsbezug auf die Ebene des individuellen Arbeitsmarktstatus, zeigt sich eine beträchtliche Statusdynamik. Ganzjährige Arbeitslosigkeit oder Erwerbstätigkeit während des Leistungsbezugs tritt deutlich seltener auf als der ganzjährige Leistungsbezug selbst. Man beobachtet einen weiteren Drehtüreffekt: Es findet eine erhebliche, wechselnde Erwerbsbeteiligung innerhalb des Leistungsbezugs statt, indem die Leistungsempfänger häufig Arbeit aufnehmen und wieder beenden, wobei das in Arbeit erzielte Entgelt nicht bedarfsdeckend ist. Arbeitsaufnahmen aus dem Leistungsbezug heraus gehen nämlich nur in $51 \%$ der Fälle auch mit dem Ende des Bezugs einher (ebd.). Die Möglichkeiten, mit einer Beschäftigung den Leistungsbezug zu beenden, 
sind abhängig von individuellen Eigenschaften und dem Haushaltskontext. So zeigen Koller und Rudolph (2011), dass für Alleinerziehende und Paare mit Kindern eine Bedarfsdeckung bei Aufnahme einer sozialversicherungspflichtigen Beschäftigung nur in 37 bzw. 38 \% der Fälle vorliegt. Achatz und Trappmann (2011) berichten für Frauen mit zu betreuenden Kindern im Haushalt sowie für Haushalte, in denen Angehörige gepflegt werden, eine geringere Wahrscheinlichkeit von erwerbsbezogenen Abgängen aus der Grundsicherung.

An dieser Stelle zeigt sich eine erste Grenze der Aktivierungspolitik: Die Aktivierung und Förderung der Arbeitsmarktintegration setzt an der Person an. Bei einer gelingenden Erwerbsintegration ist aber nicht gewährleistet, dass auch der Leistungsbezug beendet wird. Neben der realisierten Arbeitszeit und dem Lohnniveau ist eine Beendigung der Bedürftigkeit abhängig vom Haushaltskontext und der dadurch bestimmten Höhe des Einkommens, das erzielt werden müsste, um den Bedarf zu decken.

Eine zweite Grenze der Aktivierungspolitik besteht darin, dass nur ein Teil der Leistungsempfänger adressiert werden kann. Die Ausgestaltung der Grundsicherung als Mindestsicherung für den gesamten Haushalt bringt mit sich, dass unter den Leistungsempfängern auch Kinder und weitere Haushaltsmitglieder sind, die wegen der Betreuung von Kindern oder pflegebedürftigen Angehörigen dem Arbeitsmarkt nicht zur Verfügung stehen. Darüber hinaus sind knapp $30 \%$ bereits erwerbstätig und $10 \%$ in arbeitsmarktpolitischen Maßnahmen. Der Kreis von als arbeitslos geführten Personen ist somit überschaubar. Nur bei diesen Personen kann eine aktivierende Arbeitsmarktpolitik ansetzen und bei der Arbeitssuche unterstützen bzw. mehr Eigeninitiative einfordern. Befragungsergebnisse zeigen unter arbeitslosen ALG-II-Empfängern keine geringere Bereitschaft Stellen anzunehmen als unter anderen Arbeitslosen (Brenke 2010). Arbeitsuchende Arbeitslosengeld-II-Empfänger weisen darüber hinaus eine tendenziell höhere Arbeitsmotivation auf als die nicht-beziehende arbeitsuchende Bevölkerung und sie würden auch eher ungünstige Arbeitsbedingungen akzeptieren (Beste et al. 2010).

Die vergleichsweise hohe Anzahl von erwerbstätigen Leistungsbeziehern und die Aufnahmen von nicht bedarfsdeckender Beschäftigung sprechen des Weiteren dafür, dass die Höhe der Transferleistungen als impliziter Mindestlohn sowie die hohe Transferentzugsrate bei Erwerbseinkommen nicht die Anreize für eine Erwerbstätigkeit eliminieren.

\section{Zusammenfassung und Fazit}

Die Einführung der Grundsicherung für Arbeitsuchende war mit der Erwartung verbunden, dass durch eine verstärkte Anwendung des Prinzips des „Förderns und Forderns“ die Bedürftigkeit reduziert wird. Die relativ hohe Dauer und der hohe Bestand an Leistungsberechtigten im Aggregat sprechen zunächst dafür, dass diese Ziele nicht erreicht wurden. Wir haben ein genaueres Bild des Bezugs von Leistungen der Grundsicherung für Arbeitsuchende nach SGB II gezeichnet. Die präsentierten Befunde werfen ein etwas anderes Licht auf das System der Grundsicherung und deren Betroffene, welches aber nicht weniger problematisch ist. Zwar gibt es Ansatzpunkte für Förderungen auf individueller Ebene, um Hemmnissen für einen erwerbsbezogenen Abgang aus der Grundsicherung (Achatz/Trappmann 2011) zum Beispiel durch Qualifizierung zu begegnen. Dies wird aber von anderen Prozessen, die weniger am Individuum festzumachen sind, deutlich überlagert.

Zum einen ist hier der Haushaltskontext zu nennen, der aufgrund der Logik des Systems der Grundsicherung als bedarfsgeprüfte Haushaltsleis- tung zum Tragen kommt, indem er einerseits die Einkommensschwelle insbesondere in Mehrpersonenhaushalten so hoch legt, dass eine individuell erreichte Arbeitsmarktintegration in der Mehrzahl der Fälle nicht zu einem Ende des Leistungsbezugs führt. Andererseits schränken Betreuungsaufgaben im Haushalt die Verfügbarkeit der Haushaltsmitglieder für den Arbeitsmarkt sowie den möglichen Erwerbsumfang ein. Zum anderen scheint der Arbeitsmarkt für Personen aus ALG-II-Haushalten häufig nur instabile und niedrig entlohnte Beschäftigung bereitzuhalten.

Dieses Zusammenspiel von Haushaltskontext, dem Verhältnis von erwerbsfähigen zu nicht-erwerbsfähigen Leistungsberechtigten und den ungünstigen Arbeitsmarktbedingungen führt dazu, dass die einzelnen Leistungsbezugsperioden lange andauern können. Darüber hinaus hat sich gezeigt, dass die Überwindung der Bedürftigkeit häufig nicht nachhaltig ist. Dies kann demnach auf der Ebene einzelner Haushalte zu durchaus beträchtlichen kumulierten Phasen der Bedürftigkeit führen.

Dass das Ziel der Reduzierung der Bedürftigkeit nicht erreicht werden konnte, kann somit in den meisten Fällen nicht den Individuen zugeschrieben werden. Dies wird nochmals durch die zusätzliche Dynamik innerhalb des Systems unterhalb der Bedürftigkeitsgrenze unterstrichen. Es finden deutlich häufiger Statuswechsel zwischen Arbeitslosigkeit und Erwerbstätigkeit statt als sich in den Ausstiegen aus dem Leistungsbezug ausdrücken. Auf der Ebene der erwerbsfähigen Individuen ist Leistungsbezug nicht mit Erwerbslosigkeit gleichzusetzen - durch eine zumindest temporäre Arbeitsmarktpartizipation wird das Risiko sozialer Exklusion abgemildert.

\section{LITERATUR}

Achatz, J./Trappmann, M. (2011): Arbeitsmarktvermittelte Abgänge aus der Grundsicherung. Der Einfluss von personen- und haushaltsgebundenen Barrieren, IAB-Discussion Paper 2/2011, Nürnberg

Bergdolt, R./Fritz, J./Hartmann, M./Hofmann, B./Pospech, I. (2010): Grundsicherung für Arbeitsuchende: Verweildauern von Hilfebedürftigen, Statistik der Bundesagentur für Arbeit, Nürnberg

Bergdolt, R./Fritz, J./Hartmann, M./Hofmann, B./Pospech, I. (2013): Verweildauern von Leistungsberechtigten in der Grundsicherung für Arbeitsuchende, Methodenbericht, Statistik der Bundesagentur für Arbeit, Nürnberg

Beste, J./Bethmann, A./Trappmann, M. (2010): Arbeitsmotivation und Konzessionsbereitschaft: ALG-II-Bezug ist nur selten ein Ruhekissen, IAB-Kurzbericht 15/2010, Nürnberg

Brenke, K. (2010): Fünf Jahre Hartz IV - Das Problem ist nicht die Arbeitsmoral, DIW-Wochenbericht 77 (6), Berlin

Bruckmeier, K./Eggs, J./Himsel, C./Trappmann, M./Walwei, U. (2013):

Aufstocker im SGB II: Steinig und lang - der Weg aus dem Leistungsbezug, IAB-Kurzbericht 14/2013, Nürnberg

Christoph, B./Lietzmann, T. (2013): Je länger, je weniger? Zum Zusammenhang zwischen der Dauer des ALG-II-Leistungsbezugs und den materiellen Lebensbedingungen der Betroffenen, in: Zeitschrift für Sozialreform 59 (2), S. $167-196$

Dingeldey, I. (2006): Aktivierender Wohlfahrtsstaat und sozialpolitische Steuerung, in: Aus Politik und Zeitgeschichte (8-9), S. 3-9

Eichhorst, W./Grienberger-Zingerle, M./Konle-Seidl, R. (2010): Activating labor market and social policies in Germany: From status protection to basic income support, in: German Policy Studies 6 (1), S. 65-106

Franz, W. (2006): Bewertung Hartz IV und Ein-Euro-Jobs durch den Sachverständigenrat, in: Zeitschrift für Wirtschaftspolitik 55 (2), S. 177-181 Giddens, A. (1997): Jenseits von Links und Rechts. Die Zukunft radikaler Demokratie, Frankfurt a. M.

Giddens, A. (1999): Der dritte Weg. Die Erneuerung der sozialen Demokratie, Frankfurt a. M.

Graf, T./Rudolph, H. (2009): Dynamik im SGB II 2005-2007: Viele Bedarfsgemeinschaften bleiben lange bedürftig, IAB-Kurzbericht 5/2009, Nürnberg 
Habich, R./Headey, B./Krause, P. (1991): Armut im Reichtum - Ist die Bundes republik Deutschland eine Zwei-Drittel-Gesellschaft?, in: Rendtel, U./Wagner, G. (Hrsg.): Lebenslagen im Wandel: Zur Einkommensdynamik in Deutschland seit 1984, Frankfurt a. M./New York

Kaltenborn, B./Schiwarow, J. (2006): Hartz IV: Deutlich mehr Fürsorgeempfängerinnen und Fürsorgeempfänger, Blickpunkt Arbeit und Wirtschaft 5/2008, Berlin

Koller, L./Rudolph, H. (2011): Arbeitsaufnahmen von SGB-II-Leistungsempfängern: Viele Jobs von kurzer Dauer, IAB-Kurzbericht 14/2011 Nürnberg

Leibfried, S./Leisering, L./Buhr, P./Ludwig, M./Mädje, E./Olk, T./Voges, W./

Zwick, M. (1995): Zeit der Armut. Lebensläufe im Sozialstaat, Frankfurt a. M. Rudolph, H./Graf, T./Koller, L./Lietzmann, T (2013): Anhang B2: Das Administrative Panel (AdminP) - Forschungsdaten mit Haushaltskontext zum SGB II, in: Dietz, M./Kupka, P./Ramos Lobato, P. (Hrsg.): Acht Jahre Grundsicherung für Arbeitsuchende. Strukturen - Prozesse - Wirkungen, Bielefeld, S. 351-357 Sinn, H.-W./Holzner, C./Meister, W./Ochel, W./Werding, M. (2002): Aktivierende Sozialhilfe. Ein Weg zu mehr Beschäftigung und Wachstum, ifo Schnelldienst 9/2002, München

Sinn, H.-W./Holzner, C./Meister, W./Ochel, W./Werding, M. (2006): Aktivierende Sozialhilfe 2006: Das Kombilohn-Modell des ifo Instituts, ifo Schnelldienst 2/2006, München

Statistik der Bundesagentur für Arbeit (2010): Analyse der Grundsicherung fü Arbeitsuchende im Dezember 2010, Nürnberg

Statistik der Bundesagentur für Arbeit (2011): Analyse der Grundsicherung für Arbeitsuchende im Dezember 2011, Nürnberg

Statistik der Bundesagentur für Arbeit (2013): Analyse der Grundsicherung für Arbeitsuchende im Juli 2013, Nürnberg

Statistik der Bundesagentur für Arbeit (2014a): Analyse der Grundsicherung für Arbeitsuchende im Januar 2014, Nürnberg

Statistik der Bundesagentur für Arbeit (2014b): Statistik der Grundsicherung für Arbeitsuchende nach dem SGB II, Zeitreihe Eckwerte SGB II nach Ländern, April, Nürnberg

\section{AUTOREN}

LENA KOLLER-BÖSEL, Dr., ist wissenschaftliche Mitarbeiterin in der Forschungsgruppe "Dynamik in der Grundsicherung" am Institut für Arbeitsmarkt- und Berufsforschung der Bundesagentur für Arbeit (IAB), Nürnberg. Arbeitsschwerpunkte: Armutsforschung, Lohn- und Statusmobilität von SGB-II-Leistungsempfängern.

lena.koller-boesel@iab.de

TORSTEN LIETZMANN, Dipl. Soziologe, ist stellvertretender Leiter der Forschungsgruppe "Dynamik in der Grundsicherung" am IAB. Arbeitsschwerpunkte: Armutsforschung, Familiensoziologie.

torsten.lietzmann@iab.de

HELMUT RUDOLPH, Dipl. Mathematiker, war Leiter der Forschungsgruppe "Dynamik in der Grundsicherung" am IAB. Arbeitsschwerpunkte: Dynamik in der Grundsicherung, Aufstocker im SGB II, atypische Beschäftigung.

@elmut.rudolph@iab.de 